\title{
Criterios de transferibilidad del enfoque concurrente en los procesos de diseño y desarrollo de productos de las pequeñas y medianas empresas chilenas
}

\author{
Transferability criteria of the concurrent approach in the product design and \\ development processes of Chilean small and middle size companies
}

\author{
Carole Baudin ${ }^{1}$
}

Recibido 24 de diciembre de 2009, aceptado 11 de enero de 2011

Received: December 24, 2009 Accepted: January 11, 2011

\begin{abstract}
RESUMEN
Este artículo presenta los resultados de la primera fase de un estudio que pretende generar un modelo de evaluación y mejoramiento de los Procesos de Diseño y Desarrollo de Productos (PDDP) de las pequeñas y medianas empresas (PyMEs) manufactureras chilenas basado en el enfoque concurrente a fin de insertarlas a los mercados competitivos actuales. En efecto, muchos autores demostraron que, en nuestra sociedad globalizada, la capacidad de una empresa en tener una buena conducción y desarrollo de las fases de diseño de productos determina una gran parte su eficacia industrial. En particular, los modelos basados en la Ingeniería Concurrente (IC) parecen ser los más apropiados para responder rápida y adecuadamente a mercados siempre más exigentes. Sin embargo, existen pocos estudios que demuestren la aplicabilidad de estos modelos a las PyMEs. Esta primera fase pretende entonces establecer criterios de transferibilidad del enfoque concurrente en pequeñas y medianas empresas, analizando el corpus teórico y experimental relativo a la Ingeniería Concurrente (IC) y su aplicación en PYME, contrastando estos análisis con los primeros resultados de una investigación explorativa y cualitativa de tres PyMEs manufactureras chilenas. Estos criterios de transferibilidad se basan por una parte sobre una interpretación del enfoque concurrente al analizar la literatura existente y los patrones de comportamiento de las PyMEs para así destacar las dimensiones críticas particulares, por otra parte, se basa en un análisis del tipo de modelo de intervención que permita su implementación en este tipo de empresas.
\end{abstract}

Palabras clave: Procesos de diseño y desarrollo de productos (PDDP), ingeniería concurrente, pequeñas y medianas empresas (PyMEs), modelo de mejoramiento, tablas de madurez.

\section{ABSTRACT}

The results of the first stage of a study aimed at generating a model for the evaluation and improvement of Product Design and Development Processes (PDDP) of Chilean small and middle size enterprises (SMEs) based on the concurrent approach with the aim of inserting them in current competitive markets are presented. Many authors have shown that in our globalized society the ability of a company to provide good guidance and development of the product design stages determines largely its industrial efficiency. In particular, models based on Concurrent Engineering $(C E)$ seem to be the most appropriate to respond rapidly and adequately to ever more demanding markets. However, there are few studies showing the applicability of these models to SMEs. Therefore this first stage is aimed at establishing transferability criteria of the concurrent approach in small and middle size companies, analyzing the technical and experimental corpus related to CE and its application in SMEs, contrasting these analyses with the first results of an exploratory and qualitative investigation of three Chilean manufacturing SMEs. These transferability criteria are based on the one hand on an interpretation of the concurrent approach from the existing literature and the performance pattern of the SMEs in order to highlight the particular critical sizes, and on the other hand on an analysis of the type of intervention model that will allow its implementation in these kinds of companies.

Keywords: Product design and development processes (PDDP), concurrent engineering, small and middle size enterprises (SMEs), improvement model, maturity grid.

1 Departamento de Tecnologías Generales. Facultad Tecnológica. Universidad de Santiago de Chile (USACH). Avda. Ecuador \#3769, Estación Central. Santiago, Chile.E-mail: carole.baudin@usach.cl 


\section{INTRODUCCIÓN}

\section{Los Procesos de Diseño y Desarrollo de Productos}

El proceso de diseño y desarrollo de productos (PDDP) comprende "una serie de actividades que comienzan con la gestación de una idea innovadora y continúan con el desarrollo del concepto en sí mismo. Algunas empresas disponen de un proceso formalmente definido, mientras que en otras organizaciones el mismo es de carácter informal y por ello no lo conocen o no lo pueden identificar y definir" [23].

Si bien algunos autores llevan las fronteras de este proceso hasta la puesta en mercado de un producto, se entenderá, en este trabajo, el PDDP como el proceso que parte desde una idea de producto o un encargo, hasta la fabricación del prototipo.

Se pueden observar en la bibliografía dos tipos de interpretación de este proceso. El primero, extraído de las ciencias de la ingeniería $[1,6,20]$, entiende este proceso como todas aquellas actividades relacionadas a la gestión de desarrollo de nuevos productos en una empresa. En este caso, tienen que ver con la conducción de proyecto de diseño de un nuevo producto. El segundo, desde las recién proclamadas "ciencias del Diseño" $[6,8,12]$, considera los procesos de diseño como todo tipo de actividad de creación (artística, industrial, arquitectural, etc.) y lo asimila al proceso cognitivo de creación. Llevado a la manufactura, este proceso de diseño describe una sucesión de actividades técnicas que llevan a la creación de un producto, enfocándose en la generación, evaluación e implementación de soluciones [18].

La ambición del trabajo aquí presentado es acoger estas dos interpretaciones, al entender de manera más global el PDDP, al igual que Maier y sus colaboradores como un proceso técnico y social complejo [16], considerando tanto el proyecto como el proceso para llegar al producto. De esta forma, la metodología investigativa empleada debe adecuarse a esta naturaleza compleja, privilegiando un enfoque sistémico y articulando un approach operacional y cualitativo permitiendo recoger las dimensiones tanto técnicas como sociales. Es así que el estudio contempla tanto un riguroso análisis de los modelos propuestos en cada una de estas perspectivas, como una investigación explorativa, cualitativa y comprensiva de los PDDP en tres empresas chilenas.

Por otra parte, y a pesar de las diferencias de interpretación del PDDP descritas, se encuentra un consenso sobre la importancia de optimizar los procesos de diseño y desarrollo de productos para las empresas. Desde las ciencias del Diseño, se ha demostrado la rentabilidad de un "buen diseño" (Design Performance) al mostrar la relación entre el mejoramiento continuo del diseño de productos y los buenos resultados comerciales de las empresas, y, en particular, de las PyMEs [18]. De la misma forma, pero desde la perspectiva de gestión, se ha demostrado que una estrategia basada únicamente en reducción de costos en búsqueda de una mayor competitividad empresarial no es suficiente para las empresas actuales, y no permite responder a las variaciones de demanda del mercado [18]. Es así que los esfuerzos deben enfocarse sobre los procesos de diseño de nuevos productos. Desde la ingeniería, Moges [17], demuestra a través del modelo de la gran firma Lexmark, cómo el optimizar la fase de diseño -y, en particular las fases de diseño conceptual-permite a las empresas reducir sus costos y responder más rápidamente al mercado.

Asimismo, la literatura ha aportado evidencias significativas de que la optimización de los procesos de diseño y desarrollo de productos de una empresa es una estrategia potente de eficacia empresarial, tanto para las grandes empresas [17] como las PYMEs [18].

\section{Del enfoque Concurrente para optimizar los PDDP de las PYMEs}

Uno de los modelos de optimización de los PDDP internacionalmente reconocido hoy por su eficiencia es el modelo de la Ingeniería Concurrente (IC). La IC, como se presentará en el siguiente párrafo, es entendida tanto como un enfoque, una filosofía, y como una metodología con una amplia gama de métodos, técnicas y tecnologías asociadas, tanto blandas como duras.

Cabe destacar que una gran parte de la literatura existente al respecto enfatiza y trabaja la metodología de la IC, y sus diversos instrumentos y tecnologías asociados. Una primera hipótesis de este trabajo, que se valida con el respaldo de distintos trabajos de implementación de la IC en PyMEs, plantea que la 
implementación de la IC como metodología con su arsenal de técnicas y tecnologías no se adapta a las realidades de las PyMEs. No obstante, se plantea que la IC vista como enfoque puede ser adecuada para este tipo de empresas, porque permite optimizar los PDDP tanto en su nivel de gestión de proyecto como en la optimización del producto resultado de este, por lo que se adecua con la visión global del PDDP propuesta anteriormente.

Es así que la primera parte de este artículo, después de una descripción contextualizada del modelo de la IC, y después del análisis de trabajos presentando su aplicación en PyME, intenta establecer los requerimientos fundamentales para la transferibilidad del enfoque concurrente en PyMEs. En la segunda parte de este artículo, y entendiendo que la transferibilidad del enfoque concurrente depende de los modelos de su implementación, se analizarán varios modelos de intervención de los PDDP en PYME a fin de esbozar una propuesta de modelo de evaluación y mejoramiento de estos procesos basado en el enfoque concurrente.

\section{MODELO DE INGENIERÍA CONCURRENTE}

\section{Ingeniería Concurrente: Historia y Definición}

La definición de la ingeniería concurrente (IC) así como su nacimiento tienen numerosas versiones en la literatura. Esto se puede interpretar por el hecho de que su emergencia se debe a un conjunto de esfuerzos dispersos que han dado luz tanto a técnicas $\mathrm{y}$ herramientas como a un paradigma global en la dinámica de diseño y desarrollo de productos.

Es así que según Horacio Ahuett [3], la IC, si bien fue declarada como paradigma a partir de los años 80 , emergió décadas antes a través de técnicas que hoy forman parte de la metodología de la IC, y se cristalizó como respuesta a las problemáticas de Productividad y Calidad que se plantearon en los sistemas industriales a partir de la segunda guerra mundial. Asimismo para Ahuett el paradigma de la IC era ya latente con la venida de los conceptos de Just in Time (JIT) y se inició efectivamente con el concepto de Diseño Robusto (RD), introducido por Taguchi para la Nippon Telegraph and Telephone (NTT) "a fin de optimizar el producto desde el punto de vista de la fabricación". Luego, en los años 70, dos profesores japoneses desarrollan otra de las técnicas fundamentales de la IC: el Despliegue de la Función de Calidad (QFD), enfocado a la optimización de la calidad del producto y de sus procesos de fabricación, en miras de responder óptimamente a los requerimientos del cliente. Esta técnica fue utilizada por la firma Mitsubishi Heavy Industries y rápidamente exportada en otros países.

En el mismo periodo, Honda introduce el concepto de Ventas, Ingeniería y Desarrollo (SED, sigla en inglés: Sales, Engineering, Development) en sus procesos de desarrollo de productos a fin de ser capaz de "responder con mayor rapidez a los deseos del cliente y a los movimiento de la competencia. El sistema SED se basaba en la formación de equipos de diseño, integrados por personal de ventas, ingeniería y desarrollo, es decir, formaba equipos multidisciplinarios para el desarrollo de productos" [3]. Esta iniciativa fue el preámbulo de la IC como nuevo paradigma. Tiempo después, el nombre de Ingeniería Concurrente aparece en la firma Nissan, en 1987, que adoptó el término de "IC consciente", para distinguirlo de la IC que por 30 años habían aplicado de manera "inconsciente". A partir de este momento, el término y metodología se extendió" a nivel internacional [3].

Para concluir este breve recorrido histórico, De Castro y Gimenez [5] constatan que la IC se impone hoy como nuevo paradigma tanto organizacional como operativo, y surge como evolución natural de las organizaciones a fin de responder de manera eficiente a los nuevos escenarios complejos y competitivos que experimentan. En efecto, la IC surge como respuesta a la necesidad de las empresas de responder rápida y adecuadamente a las exigencias de los mercados. Esto supone para las empresas reducir siempre más sus tiempos de diseño y producción y satisfacer los clientes.

La IC propone una alternativa a los procesos tradicionales de diseño y desarrollo de productos que siguen un modelo secuencial donde cada actor trabaja de manera independiente y aislada, e interviniendo de manera cronológica en los procesos, siguiendo así una organización por función donde la toma de decisión es fundamentalmente jerárquica $[11,20]$.

Ante esta linealidad de los procesos tradicionales, la ingeniería concurrente (IC) propone "un enfoque 
sistemático para el diseño paralelo e integrado de productos y los procesos relacionados, incluyendo manufactura y servicios de apoyo, con la intención de que los desarrolladores consideren, desde el inicio del proyecto, todos los elementos del ciclo de vida del producto, desde su concepción hasta su eliminación y reciclaje, incluyendo calidad, costo, planeación y requerimientos del usuario" [2].

La especificidad de la ingeniería concurrente en los procesos de diseño es que permite la realización simultánea de todas las tareas de desarrollo hasta la fabricación del prototipo, permitiendo así evitar re-procesos, mientras que en un modelo secuencial sólo existe la posibilidad de volver a las fases anteriores de desarrollo del producto [22].

Basado en estudios empíricos que han demostrado que el $60 \%$ del costo de fabricación del producto se fija en la fase de diseño [22], la ingeniería concurrente privilegia, en particular, las fases de especificación y diseño de producto, a fin de obtener una definición más completa y refinada, que reduzca la necesidad de cambios posteriores, y así racionaliza los tiempos y recursos de proyecto de diseño. Además, establece un desarrollo simultáneo del producto integrando el punto de vista de fabricación y procesos, control de calidad y marketing generando así un tiempo más corto en el desarrollo de nuevos productos. Los casos experimentales han demostrado que la reducción del tiempo varía en función del tipo de producto, pero de forma general se han identificado reducciones que van del $25 \%$ al $50 \%$ en el tiempo total de desarrollo y producción [22].

Actualmente, la IC está reconocida y aplicada principalmente a través de la batería de técnicas y tecnologías tanto blandas como duras que apoyan la IC.

Como se desprende de su historia, aplicación y desarrollo actual, la IC como metodología responde preferentemente a problemáticas relativas al diseño y desarrollo de productos complejos generalmente llevados en el seno de organizaciones grandes y complejas. Asimismo, su transferibilidad en empresas de pequeño y mediano tamaño ha sido muy poco abordada, y la naturaleza de los estudios realizados trata de avanzar en la generación de nuevos modelos algorítmicos capaces de soportar la comunicación de estas empresas en entornos concurrentes [7, 9], o bien aborda la implementación de la IC en las PyMEs, pero considerándola desde su enfoque metodológico y tecnológico, por tanto sujeta a las capacidades y compromisos en términos de recursos financieros, humanos y técnicos de cada PyME.

Es así que en uno de sus primeros estudios, Luna [14] mostró que si bien muchas de las 35 PyMEs manufactureras estudiadas conocían e incluso aplicaban algunas de las tecnologías asociadas a la IC, pocas tenían conocimiento de la filosofía concurrente. Este estudio mostró también que, en caso de aplicar alguna tecnología de la IC, estas empresas solían aplicar tecnologías blandas (como QFD, TRIZ, DFMA, A.V., método Kanseï, CTC, etc.) más que duras (como CAD/ CAM / CAE/ CNC o PDM) [14, 21], considerando estas últimas como una alta inversión, representando así una barrera a la implementación de la IC. Por fin, otro estudio de Luna [13] constata que cuando las empresas integran tecnologías duras no logran integrarlas de manera eficiente a sus PDDP. Este estudio desvela una tendencia de las PyMEs que permite plantear que la IC como paradigma o enfoque, y no como instrumento es transferible a las PyMEs y permitiría mejorar los PDDP de estas. Esta afirmación implica poder describir qué es el enfoque concurrente.

\section{Hacia la definición de un enfoque concurrente}

Más allá de su dimensión instrumental, la IC presenta un nuevo paradigma en la dinámica del proceso de diseño y desarrollo definido de distintas maneras según los autores.

Para Luna, la IC propone una "filosofía de trabajo basada en sistemas de información y fundamentada en la idea de convergencia, simultaneidad o concurrencia de la información contenida en todo el ciclo de vida de un producto sobre el diseño del mismo" [15].

Es así que se diferencia de los modelos tradicionales de diseño y desarrollo, introduciendo una relación estrecha entre la ingeniería de producto y la ingeniería de procesos en cada fase del proceso de diseño. De esta forma, la IC abarca la relación, en cualquier nivel en que ocurra, entre todo tipo de recursos (personas, material, maquinaria, tecnología, información, etc.) de los que dispone una organización [13]. 
En esta perspectiva, el enfoque concurrente aplicado al producto supone una concurrencia, es decir, una integración o retroalimentación de información desde áreas de fabricación, ensamble, distribución, etc., hacia el diseño del objeto, esto es, que permite diseñar al mismo tiempo el producto y el sistema de fabricación del producto. Aplicado a la gestión de proceso o planificación, el concepto de concurrencia supone una simultaneidad de tareas y una organización flexible.

Por otra parte, a nivel operativo, este enfoque está basado en la mejora a través de la formación de equipos de trabajo multidisciplinarios, del intercambio de información y conocimiento y de la aplicación de tecnologías y herramientas y orientada a la satisfacción del cliente.

La IC “apoya los valores de trabajo en equipo multidisciplinario como la cooperación, la confianza, el compartir y el intercambio de información y conocimiento; de tal manera que la toma de decisiones durante la etapa de diseño considere simultáneamente todos los aspectos del Ciclo de Vida de Productos" [13].

Según Ríos [22], la aplicación de métodos de la IC se basa en cinco acciones fundamentales:

- Trabajo en equipos multidisciplinarios con participación de proveedores.

- Especificación de detalle del producto, desde el punto de vista de ingeniería, a partir de los términos definidos por el cliente.

- Especificación de los parámetros que permiten asegurar la optimización de la calidad del producto.

- Optimización del diseño del producto, teniendo presente todos los aspectos que afectan a su ciclo de vida: funcionalidad, fabricación, montaje, mantenimiento y servicio, reciclaje, retirada, etc.

- Desarrollo simultáneo del producto, equipo de fabricación y procesos, control de calidad y marketing.

Se desprende de estas definiciones que el intercambio e integración de información, así como la interdisciplinariedad son clave para el fomento de un enfoque concurrente. No obstante, las visiones anteriormente descritas consideran estos dos items, dentro de una perspectiva operacional de la IC, correspondiendo a una visión estrictamente ingenieril de los PDDP. Otros autores, entendiendo el proceso de diseño y desarrollo como proceso cognitivo y social, lo contemplan desde la perspectiva de transferencia del conocimiento. Asimismo, para Nicolini [19] interpretando la teoría de Nonaka en gestión del conocimiento, considera el PDDP como motor innovación, y por lo tanto, motor de aprendizaje para las empresas suponiendo desarrollar un espacio organizacional y de diálogo donde puedan revisarse los supuestos desde los cuales la organización es pensada.

Desde esta mirada, Nicolini considera que "las metodologías concurrente, simultánea e integrada permiten obtener mejores resultados que la secuencial," en particular, porque busca "tener un lenguaje en común, tanto respecto de las distintas disciplinas que intervienen en el desarrollo de productos como en lo que se refiere a las herramientas que se puedan llegar a usar" [19]. Para este autor, esta forma de trabajo asegura un "proceso de aprendizaje, que se genera a partir de la realimentación de los conocimientos tácitos y de los conocimientos codificados que poseen tanto los integrantes de la empresa, como todos aquellos que participen del desarrollo, como ser proveedores o usuarios" [19].

Es así que aparece que el enfoque concurrente por una parte busca generar un proceso de diseño intersubjetivo, permitiendo así integrar los distintos puntos de vista de los actores que se encuentran a lo largo del ciclo de vida del producto. Esto supone que se generen espacios de intercambio de información y conocimientos, y espacios de creación de ideas. Esto supone también una organización que permita que se desarrolle el proceso de diseño a través de un grupo de personas (organización por proyecto).

Por otra parte, el enfoque concurrente busca, desde un nivel operativo, alargar las fases iniciales de análisis y definición y/ o especificaciones de diseño (que se deben generar mediante un trabajo colaborativo) a fin de evitar que se deba volver atrás una vez empezado las fases de diseño de detalle. Estos re-procesos ulteriores son los que generan una pérdida de tiempo y de dinero para las empresas. "La temprana disponibilidad de información reduce la incertidumbre y facilita la rápida detección de problemas que permiten a las 
empresas evitar el consumo excesivo de tiempo debido a los cambios propuestos [19]". Por tanto, el alargamiento de las fases iniciales debe acompañarse de herramientas de análisis y creatividad sistemática y colaborativa a fin de que estas fases sean cumplidas de manera óptima.

\section{CRITERIOS DE TRANSFERIBILIDAD DEL ENFOQUE CONCURRENTE EN PYMES}

Se entiende por transferibilidad, la capacidad de transferencia y por criterios de transferibilidad del enfoque concurrente, los elementos que permiten validar o asegurar una capacidad óptima de transferencia, o implementación del enfoque concurrente en las empresas.

Para establecer criterios de transferibilidad del enfoque concurrente corresponde en un primer tiempo, observar y entender cómo las PyMEs llevan sus procesos de diseño y desarrollo de productos a fin de identificar las problemáticas particulares relativas en este tipo de empresas la implementación del enfoque concurrente descrito anteriormente.

Análisis de los procesos de Diseño y desarrollo de productos en PyMEs

Los trabajos que se han realizado sobre los procesos de diseño y desarrollo de las PyMEs, a pesar de sus diferencias metodológicas y, por lo tanto de los criterios de análisis aplicados, han permitido identificar patrones aplicables a esta investigación y orientar la diagnosis de las empresas chilenas en estudio. En particular, se analizaron trabajos llevados en América Latina y Central para comparar empresas insertas en realidades cercanas. Pero también nos apoyamos en estudios internacionales, permitiendo así generalizar algunos criterios e identificar los criterios más propios a la localidad de los casos.

\section{a) Análisis desde una visión de la IC en PyMEs mexicanas}

Luna y sus colaboradores del Grupo de Investigación de Productividad y Competitividad del Departamento de Ingeniería Industrial de la Universidad del Norte (México) trabajan desde hace una década sobre la integración de la IC en PyMEs manufactureras de un barrio mexicano. En uno de sus estudios más reciente, realizan un diagnóstico de cinco de estas empresas basándose en dos herramientas de análisis: el IDEF0 y el HE-PDP-A [13].
El IDEF0, es una herramienta basada en el modelo S.A.D.T. (Structured Analysis and Design Technique). Permite identificar y visualizar gráficamente el conjunto de etapas y actividades que conforman el proceso de Diseño y Desarrollo de Productos de una empresa y la utilización de recursos en cada caso. Este instrumento permite en particular, según Luna, identificar las actividades llevadas de manera simultánea en este proceso. La herramienta HE-PPD-A permite evaluar el nivel de gestión de factores claves que identificaron los autores, para cumplir con un modelo concurrente. Este instrumento permite cuantificar el estado del arte de las empresas en relación a estos criterios de evaluación que se desprenden de cinco dimensiones del entorno concurrente establecidas por los autores. Estas dimensiones son: Organización, R.R.H.H., mercado, información y tecnología.

El análisis de las empresas según estas dos herramientas permitió a los autores establecer el siguiente diagnóstico:

- "Ninguna empresa dispone de herramienta para análisis de recursos y asignación de responsabilidad. (tipo IDEFO).

- Tres de cinco hacen análisis de ejecución simultanea de actividades.

- Solo una dispone de un equipo multidisciplinario siguiendo el proceso de diseño y desarrollo hasta el final, las otras lo hacen de manera temporal (no se mantiene hasta el cierre el proyecto).

- Existen debilidades en la evaluación y selección de proveedores, y no existe integración de esto en planeación y diseño.

- Ninguna reconoce ventajas de metodologías basadas en el conocimiento como el QFD o DFX.

- Todas tienen tecnologías para el diseño, solo dos lo integran adecuadamente al proceso de diseño.

Sin embargo, los autores reconocen potenciales de estas empresas para integrar un modelo concurrente, especialmente en cuanto "a sus aspectos fundamentales, como son: uso de herramientas de apoyo, tecnología, trabajo en equipos multidisciplinarios y apoyo de la dirección” [13].

Este trabajo propone alcances interesantes para el propósito de este estudio, en particular, porque las cinco dimensiones globales establecidas podrían 
corresponder a los criterios de transferibilidad que se están trabajando. Sin embargo, las dimensiones así identificadas por Luna y sus colaboradores, corresponden más bien a la perspectiva instrumental de la IC y no paradigmática porque se refieren a los recursos y mecanismos necesarios para introducir la IC como metodología con sus respectivas técnicas y tecnologías. Es así que, en su diagnóstico, se constata que pocas empresas están listas para introducir la metodología de la IC.

\section{b) Análisis cualitativo de los PDDP de PyMEs argentinas}

Con otra óptica, pero permitiendo precisar el diagnóstico anterior, Nicolini y sus colaboradores realizan un diagnóstico de los procesos de desarrollo de productos sobre un muestreo de PyMEs argentinas [19] desde una perspectiva comprensiva.

En su trabajo, estudian los procesos de diseño de estas empresas desde una metodología cualitativa, buscando la significación de estos procesos para los actores de estas organizaciones y enfrentándolo a los modelos teóricos existentes, y, en particular, el modelo de la ingeniería concurrente.

Los resultados así obtenidos ponen en relieve los siguientes patrones de comportamientos de este tipo de empresas:

- Las actividades de desarrollo de producto no circunscriben a una metodología preestablecida, no tienen objetivos bien definidos, consensuados y comunicados.

- No hay planificación sino asignación variable de los recursos por parte de los responsables según importancia del proyecto. Las empresas que tienen planificación lo deben a las exigencias del mercado.

- El PDDP se da en contexto de prácticas informales flexibles y en ocasiones caóticas, urgidas por la necesidad y los tiempos cortos de desarrollo y con las modalidades de comunicación típicas de las empresas PyME. En esta figura, observan que el proceso de desarrollo sólo combina algunas instancias formales.

En conclusión, estos autores constatan que "estos patrones muestran con claridad que las empresas no se ajustan a ninguna metodología predeterminada de desarrollo de productos". Para estos autores, esta afirmación motiva a reflexionar y a preguntar sobre aspectos de "índole organizativa, así como de percepción e interpretación del proceso de desarrollo de productos" [19].

Esta conclusión apunta en la dirección de la hipótesis anteriormente planteada que dice relación con la poca transferibilidad de la IC implementada como metodología. Efectivamente, al identificar la problemática de la informalidad de los procesos en las PyMEs estudiadas, como una de las características de estas empresas, Nicolini y sus colaboradores cuestionan sobre la factibilidad de introducir metodología preestablecida en sistemas donde reina la informalidad y el sentido de urgencia.

Estos autores apuntan aquí a características de las PyMEs que trascienden las fronteras latinoamericanas $\mathrm{y}$ trascienden los sectores industriales, puesto que en otro estudio llevado en Reino Unido, Filson y Lewis [10], también identifican patrones similares de comportamiento en empresas inglesas.

\section{c) Análisis de la implementación de procedimientos concurrentes en PyMEs inglesas}

Filson y Lewis [10] realizaron un estudio de sumo interés cuyo objetivo era la implementación de "procedimientos concurrentes" de diseño y desarrollo de productos en PyME inglesa. Los resultados de este trabajo muestran, por una parte, patrones muy cercanos a los identificados anteriormente en las PyMEs mexicanas y argentinas, por otra, pone en relieve una problemática fundamental de la implementación de la metodología de IC: la problemática cultural.

En un primer recorrido de los procedimientos de varias empresas locales, Filson y Lewis constatan que la mayoría de estas desarrollan sus productos desde un enfoque secuencial y no concurrente. Que los proyectos que se desarrollan son todos fuera de plazos, que la mayoría de estos proyectos no tienen planificación previa y llegan en cualquier momento; que existen pocas estructuras de comunicación entre los distintos departamentos; que nadie asume la propiedad del proyecto, aunque se admite responsabilidad.

En una segunda etapa, estos autores analizan una prueba de implementación de un procedimiento concurrente de diseño y desarrollo de productos en una PyME del rubro electrónico. Cabe destacar 
que esta implementación se realiza solamente sobre dos proyectos de la empresa, dejando el resto de los proyectos desarrollándose de manera tradicional. En este marco, los autores identifican los siguientes frenos y problemáticas relativos a la implementación de un procedimiento concurrente:

- Falla en el control de la selección de proyectos: no se realiza de acuerdo a metas y estrategias corporativas.

- Existencia de conflictos de metas /objetivos entre los distintos actores del proceso.

- Prevalencia de requerimientos de tiempos cortos: existe siempre una urgencia en entregar un producto a un cliente en un tiempo corto (debido según los autores a una planificación de venta mensual). Se responde siempre a proyectos de corto plazo (desde venta) a fin de alcanzar cifras mensuales, lo que viene a perturbar la implementación de un proceso un poco más largo en su fases conceptuales. El caso estudiado permitió en particular poner esta dimensión en relieve, puesto que se realizó una implementación aislada y gradual de un procedimiento concurrente durante la cual se insertaban proyectos urgentes.

- Falta de compromiso con el nuevo procedimiento: si bien la prueba se realizó con la aprobación de la empresa entera, el compromiso de un procedimiento de largo plazo fue rápidamente desplazado por proyectos de corto plazo.

Para concluir, este estudio arrojó que las principales problemáticas de las PyMEs en cuanto a la implementación de nuevos procedimientos de diseño y desarrollo de productos se cristalizan en particular sobre su renuencia al cambio, una actitud reactiva preponderante, y el cambio constante de prioridades, prefiriendo responder a requerimientos de corto plazo y viendo como ineluctable las respuestas reactivas, lo que genera sobrecargas de trabajo y fomenta el exceder los plazos [10].

Estos diferentes estudios permiten destacar patrones similares de las PyMEs en cuanto a sus procesos de diseño y desarrollo de productos que traspasan la variabilidad de contextos en los cuales están insertas, puesto que reencontramos características similares en empresas latinoamericanas como empresas europeas. No obstante, de acuerdo a los resultados del estudio de Filson y Lewis, aparece que el término cultural es fundamental a la hora de implementar nuevos procedimientos. El término cultural en este caso define "una combinación de valores de gestión, expectaciones y preferencias de cómo debería comportarse la empresa" [10], por lo que cambia de una empresa a otra. Dicho de otra manera, si bien las PyMEs tienen características similares por ser organizaciones de cierto tipo, sus comportamientos frente a la implementación de nuevos modelos de diseño y desarrollo de productos están determinados por características variables de sus culturas corporativas particulares.

\section{Primeras observaciones de las PyMEs chilenas}

En paralelo al análisis del corpus teórico y experimental relativo al enfoque concurrente y su aplicación en PyMEs, se realizó un estudio explorativo de los PDDP de tres PyMEs manufactureras de la Región Metropolitana de Chile. Estas empresas fueron seleccionadas por su representatividad en cuanto a tres dimensiones: 1 - nivel de complejidad de sus productos y de sus procesos de producción; 2- tamaño; 3- Historia y cultura corporativa. Adicionalmente, estas tres empresas fueron retenidas por integrar diseñadores industriales, ya sea en forma permanente o temporal.

La investigación realizada con estas empresas consistió, por una parte, en la aplicación de entrevistas abiertas y guiadas a actores clave de los PDDP. Por otra, contempló la observación participante in situ, realizadas por estudiantes en diseño industrial, durante a lo menos un mes en cada una de las empresas. La metodología empleada tiene como objetivo entender cómo se construyen los PDDP en estas empresas, y, en particular, identificar las dimensiones culturales de la construcción de estos procesos a fin de establecer los posibles frenos o dimensiones críticas de la implementación del enfoque concurrente. Si bien el tratamiento de la información así recolectada no ha sido todavía realizado, las primeras constataciones permiten esbozar algunos patrones de comportamiento.

Asimismo, se observa en una primera instancia, una relación muy variable con respecto a los procesos de diseño y desarrollo de productos. Efectivamente, de las tres empresas, una no tiene conciencia de realizar un proceso de diseño y/o desarrollo de productos, las dos restantes, si bien tienen identificado un proceso de diseño y desarrollo en su quehacer, cada una lo valoriza de manera distinta porque las dos 
lo restringen a una sola etapa del proceso global. Esta percepción e interpretación de sus procesos es de suma importancia, en la medida que si no existe valorización de estos procesos, difícilmente se podrán introducir cambios.

En una segunda instancia, y de manera general se desvelan algunas características comunes a las tres empresas. Es así que se constató que:

- El sentido de urgencia es preponderante. El desarrollo económico de las empresas está relacionado a la capacidad de responder de manera reactiva a demandas del mercado lo que deja poco espacio para establecer procedimientos donde se debe invertir tiempo para responder.

- No se desprenden etapas claras en los procesos de diseño, no existe ninguna sistematización de los procedimientos. Solo los procesos de fabricación tienen asignación de personas y a veces de tiempo.

- La innovación de producto es básicamente incremental y responde a encargos o requerimientos de mercado. De hecho, la innovación es llevada de forma reactiva (sobre propuesta o encargo). Por lo que la etapa de especificación de diseño casi no existe, y cuando existe está realizada por una sola persona, y de manera muy informal.

- Son organizaciones en la mayoría muy jerárquicas con un rol fuerte del dueño o gerente a su vez es afectiva por el tipo de interrelaciones que se generan en estas empresas que son de tipo familiar. La cultura organizacional tiene por lo tanto una resonancia emocional fuerte y depende mucho del liderazgo y experiencia de este.

- Si bien no siempre se reconoce en el seno de las empresas, existen espacios de concurrencia en torno al PDDP, gracias al tamaño de estas organizaciones y la flexibilidad que genera. (Esto se cruza con una ubicación geográfica, una unidad territorial que facilita la interacción). Sin embargo estos espacios no son formalizados y no permiten la sistematización de procedimientos.

- Existe un alto nivel de burocracia impulsado por las formas de relacionarse al otro y la institucionalidad local. En particular, existe una desconfianza ante los proveedores que impide una concepción colaborativa y asociativa.
- A pesar de esfuerzos de racionalización de procesos realizados por la mayoría (en particular, dos de tres empresas están implementando la norma ISO 9000), estos siguen una informalidad operativa que puede parecer, en un primer análisis, caótica, y por tanto precaria.

- Si bien pueden existir esfuerzos y capacidad de inversión, estos van de manera privilegiada hacia el mejoramiento del proceso de manufactura en desmedro del proceso de gestión.

En resumen, este recorrido de análisis de los PDDP en distintas PyMEs permite identificar patrones claros de este tipo de empresa que dejan suponer que las problemáticas de implementación de un enfoque concurrente no solamente tienen que ver con dimensiones estructurales de las empresas como lo son los términos de RRHH, infraestructura, capacidad tecnológica, etc., como se suelen identificar en la literatura. En estas empresas, las problemáticas residen en dimensiones más culturales que estructurales. Asimismo, la transferibilidad del enfoque concurrente en estas empresas depende de la interpretación que se hace del enfoque concurrente.

\section{Criterios para la implementación del enfoque concurrente en PyMEs}

El análisis del corpus teórico relativo a la ingeniería concurrente así como los diagnósticos de las PyMEs latinoamericanas, europeas, además de las primeras observaciones del estudio exploratorio llevado en las PyMEs chilenas permiten proponer, en esta primera fase de estudio, una interpretación del enfoque concurrente desde las capacidades que deberían presentar las empresas para poder acercarse a este enfoque. Cada una de las capacidades descritas a continuación corresponde, por una parte, a una de las dimensiones fundamentales del enfoque concurrente tal como se describe en la literatura, $\mathrm{y}$, por otra, corresponde a una de las dimensiones críticas de los PDDP de las PyMEs.

- Una capacidad de generar una estructura de proyecto (estructura flexible permitiendo crear un grupo de trabajo sobre el desarrollo de un producto) supone una capacidad de flexibilidad organizacional y recursos comunicacionales asociados (medios de comunicación, de colaboración asociados). 
- Una capacidad en dar énfasis y valorizar las etapas de definición/especificaciones de diseño del producto integrando las distintas visiones presentes en el proceso de concepción y producción de este, supone la capacidad en tener una visión compleja e integrativa del producto, capacidad en reformular el encargo $\mathrm{o}$ las necesidades que satisface el producto, $\mathrm{y}$ capacidad en generar "nudos de información" sobre el producto.

- Una capacidad en generar espacios de participación integrativa, creativa y evolutiva a todas las etapas del proceso (herramientas de interdisciplinariedad).

- Una capacidad de aprendizaje para alcanzar sistematización y racionalización de datos relativos al producto, en analizar la competencia (benchmarking) y adaptarse/posicionarse en los cambios de los entornos. Una capacidad en formalizar los saberes tácitos.

- Una capacidad comunicativa y colaborativa con las empresas satélites y recursos del tejido industrial e institucional.

- Una capacidad de visión de procesos de la empresa, donde se enfoca particularmente en los recursos necesarios en cada etapa.

El establecimiento de estos requerimientos o criterios para la implementación del enfoque concurrente en PyME es la primera fase para luego realizar un análisis sistémico y profundo de las tres empresas manufactureras chilenas en base a estos criterios y así validarlos, complementarlos, y entender los mecanismos de construcción de estos en las PyMEs.

Si bien estos criterios permiten construir un modelo de evaluación acorde a las PyMEs, es solamente la primera rama de una transferibilidad del enfoque concurrente. Esta dependerá también del tipo de modelo de intervención, o de implementación que se construirá. Asimismo, la segunda etapa de la identificación de los criterios de transferibilidad del enfoque concurrente en PyMEs dice relación con la generación de un modelo de implementación de este enfoque que esté adaptado a las características de las empresas estudiadas.

\section{MODELOS DE IMPLEMENTACIÓN}

Muchos de los estudios que han permitido analizar y diagnosticar los procesos de Diseño y desarrollo de productos de las PyMEs también proponen modelos de intervención de estos. Los modelos analizados a continuación tienen el interés de presentar modelos de implementación de la IC en empresas, y algunos, más particularmente en PyMEs.

\section{Modelo de implementación de la IC en PyMEs mexicanas}

En las PyMEs mexicanas, la metodología que propone Luna [15] es una intervención en la empresa que contempla capacitación a los directivos, acompañamiento y management de un proyecto y expansión al resto de la actividad de la empresa. Esta metodología consta de las siguientes siete etapas: Etapa 1: Introducción de la IC a las directivas de la empresa;

Etapa 2: Revisión de la Dirección: establecer una visión que integre la metodología de implementación de la IC con las políticas y plan estratégico de la empresa;

Etapa 3: Diagnóstico: Evaluar la situación actual del proceso de desarrollo de productos;

Etapa 4: Proporcionar el plan de acción que se debe seguir a lo largo del proceso de implementación;

Etapa 5: Ejecución del Proyecto: Realizar el desarrollo de producto bajo el enfoque de la IC;

Etapa 6: Retroalimentación y Mejora Continua: Lograr un proceso de mejora continua en la cual se esté monitoreando regularmente el desempeño de la IC;

Etapa 7: Expansión: extender los beneficios de la IC.

Cabe detallar más precisamente la etapa 4 de Planeación que sigue las siguientes fases predefinidas: Desarrollo del concepto, ingeniería del producto, ingeniería del proceso, producción, pruebas del producto, servicio postventa, en la cual el énfasis está sobre el manejo de técnicas como el QFD y tecnologías computacionales. La etapa 6 contempla la generación de indicadores para evaluar, monitorear y controlar los logros, y para fortalecer la metodología para permitir así su expansión en la empresa. En particular, se establecen indicadores relativos a tiempo de ejecución del proyecto y costos en la ejecución.

El modelo de implementación que propone Luna, responde a una estrategia basada en los procesos de planificación racional del proceso basado en la definición de objetivos, metas y sistemas de decisión 
formales. Si bien los resultados que entregan estos estudios son de buen augurio, no permiten demostrar la sustentabilidad de estos nuevos procedimientos en la empresa, sobre todo tomando en cuenta los patrones destacados anteriormente en este tipo de empresas, donde el sentido de urgencia vuelve de manera inexorable. Efectivamente la sustentabilidad de la implementación de la IC en las PyMEs en este caso se basa en la demostración de buenos resultados obtenidos en un caso de estudio acompañado. Sería de gran interés conocer después de los años transcurridos el nivel de implementación efectivo que han experimentado estas empresas, sin embargo, los diagnósticos anteriores nos dejan pensar que la cultura propia de las PyMEs no permite la fácil implementación de nuevos procedimientos.

Al igual que Nicolini y sus colaboradores, y considerando los diagnósticos anteriores de las PyMEs chilenas, se considera que la implementación de nuevos procesos debería basarse no en una estrategia fundamentada en procesos de planificación de metas sino que en una estrategia respondiendo a un proceso de incrementalismo lógico, o sea, donde la estrategia surge como resultante de la capacidad de aprendizaje y la experiencia. Esta perspectiva parece adecuarse más a los patrones de comportamiento de las empresas estudiadas, en particular, porque permite la gestión del cambio cultural necesaria en estas empresas.

\section{Modelos de auditoría basados en las Maturity Grid}

Las herramientas de intervención que responden a la estrategia incremental son aquellas que se basan sobre la gestión del conocimiento y aprendizaje que se ha instalado en las organizaciones en particular con los enfoques de calidad. Asimismo, en los años 90' se desarrollaron desde la gestión de calidad para las empresas instrumentos de auditoría en base a maturity grid (literalmente tablas de madurez, conocida también como tablas del conocimiento). En esta perspectiva, y considerando que la calidad de un producto depende de la calidad de sus procesos, Crosby definió una primera tabla de madurez, una tabla de calidad (QMMG-Quality Management Maturity Grid) basada en seis criterios de calidad y cinco niveles de madurez clasificados como: incertidumbre, atención, iluminación, sabiduría, certidumbre ("uncertainty, awakenning, enlightement, wisdom, certainty") [16]. Se definen los niveles de madurez como "los grados con los cuales los procesos o actividades son institucionalizados y efectivos" en una organización [18]. Evaluar así la madurez de los procesos permite "prever la habilidad de la organización para alcanzar sus metas" [18] y entrega una "guía para orientar las mejoras al describir la progresión del rendimiento mediante fases incrementales de desarrollo" [18].

Estas tablas construyen en base a la identificación, para una determinada actividad, de niveles típicos de comportamiento (niveles de madurez).

\section{a) Modelo de intervención mediante auditoría de los PDDP}

Basándose en el modelo de tabla de madurez, Moultrie y Clarkson [18] propusieron un instrumento de auditoría para optimizar los procesos de diseño de productos en PyMEs inglesas. Si bien el enfoque de estos autores no se pretende concurrente, está, sin embargo, basado en una línea de "buena práctica" de diseño, abarcando tanto la dimensión producto en sí como proceso. Resultado de una investigación exhaustiva, tanto teórica como experimental, este instrumento se divide en dos dimensiones: la evaluación del proceso de diseño (mediante cinco tablas de madurez) y la evaluación del producto diseñado (mediante siete tablas de evaluación subjetiva).

La evaluación del proceso de diseño está considerada en forma sistémica, desde un nivel macro del proceso -considerando la generación del proyecto como tal, y su gestión dentro de la empresa- hasta un nivel micro, es decir, evaluando cada actividad de la ejecución del diseño de un producto (desde las especificaciones de diseño hasta la implementación o fabricación del producto). Cada ítem (macro y micro) da lugar a una tabla de madurez. Los niveles de madurez identificados corresponden a cinco "ingredientes claves": los beneficios percibidos durante la actividad, las personas involucradas, los plazos, el nivel de formalización de la actividad y el nivel de experticia.

Cada ítem del proceso de diseño está detallado en términos de criterios de cumplir. Estos criterios son resultados del cruce de las distintas visiones teóricas relativas a "good design process" y de entrevistas de varios "awards" internacionales, y se especifican a continuación en la Figura 1. 
Por otra parte, la evaluación de los productos diseñados se realiza mediante siete tablas de evaluación subjetiva basada en las opiniones de los actores de la empresa y de los consumidores. $\mathrm{Al}$ igual que la evaluación del proceso, consta de dos niveles de análisis: un nivel de análisis macro del producto designado como la profitabilidad o rentabilidad global del producto; y un nivel más micro de las distintas dimensiones del producto que se define en seis atributos (1- calidad técnica; 2- usabilidad; 3- novedad; 4- Deseabilidad (atractividad); 5- Productibilidad; 6; utilidad), cada uno descompuestos en subatributos.

Si bien este instrumento no pretende introducir un enfoque concurrente, constatamos que el enfoque propuesto por estos autores considera el proceso de diseño tanto desde el mismo proceso como desde su resultado, el producto, de manera sistemática e integrativa. La filosofía que se desprende entonces no es muy lejana a una filosofía concurrente.

\section{MACROPROCESO O PROYECTO:}

1- Generación del proyecto:

Generación de idea y management; cultura creativa y entorno; estrategia de producto; selección de proyecto.

2- Management de proyecto:

Proceso de desarrollo de producto; gestión del riesgo; encuentros de diseñadores; gestión de objetivos e indicadores de diseño; equipos de trabajo; integración de experto diseñador.

\section{MICROPROCESO - EJECUCIÓN DE DISEÑO:}

1- Recolección de requerimientos:

Segmentación de mercado; análisis de competencia; investigación sobre necesidades del usuario; integración del punto de vista usuario; especificación de diseño

2- Diseño conceptual:

Generación conceptual; diseño estético; diseño ergonómico; evaluación y selección de conceptos

3- Implementación:

Diseño para la Manufactura y Ensamblaje; gestión del riesgo; prototipo para reducir riesgos de mercado; prototipo para reducir riesgos técnicos; evaluación

Figura 1. Criterios de evaluación del instrumento de auditoría de los PDDP para PyMEs de Moultrie y Clarkson. 2006.
Sin embargo, los criterios retenidos, en particular en cuanto al proceso de diseño no permiten fomentar una "cultura" concurrente, puesto que describen etapas del proceso, y los distintos niveles de "entregables" o resultados que deberían cumplir, sin enfocar sobre las formas de generar estas etapas.

\section{b) Instrumentos de mejoramiento de los entornos de la IC}

Otro instrumento de sumo interés en esta investigación es el propuesto por Carter y Stilwell, aquí relatado desde la traducción de Aguayo [1, 4].

El instrumento de Carter y Stiwell [4] permite diagnosticar y mejorar los entornos de la IC de una empresa.

Este enfoque supone que existen "entornos" o espacios que favorecen la implementación de los nuevos procedimientos que implica la IC. Dicho de otra forma, la implementación de la IC en una empresa afecta distintas dimensiones que son aquellas que hay que mejorar para lograr una optimización de los procesos concurrentes.

Cabe destacar que los "entornos" que describe Carter son muy cercanos al concepto de criterios de transferibilidad que se definen en este artículo, sin embargo, suponen que existan dimensiones exógenas que propicien o no la implementación de la IC; esta visión sigue siendo una visión metodológica de la IC, entendiendo estas dimensiones desde una perspectiva instrumental. En cambio, los criterios de transferibilidad son endógenos a las empresas, por lo que se eligió aquí interpretar el enfoque concurrente desde las capacidades de la empresa.

Sin embargo, donde se definieron aquí más bien capacidad, se definen en la propuesta de Carter como "factores estructurales como la organización, la infraestructura de comunicación, los requisitos y desarrollo del producto" $[1,4]$.

Cabe destacar que el modelo de Carter implica un presupuesto del autor basado en la convicción que la IC se puede aplicar a cualquier tipo de empresa [17]. De hecho, según Carter el "órgano director" que determina "el acercamiento" de una empresa a la IC no es el tipo de empresa sino el nivel de complejidad del producto desarrollado. Es así que para Carter, existen cuatro enfoques distintos del proceso de diseño y desarrollo de productos y cada 
enfoque es correlativo de un nivel de complejidad de los productos realizados. De este modo, define cuatro tipos de procesos correspondientes a enfoque de Tarea, de Proyecto, de Programa, de Empresa. Cada uno de estos niveles puede integrar procesos concurrentes con niveles distintos de acercamiento o "puntos de equilibrio" $[1,4]$.

El instrumento que propone Carter se compone de cuatro herramientas:

1- Una encuesta de valoración de la empresa realizada respecto a las cuatro dimensiones estructurales de la IC (organización, la infraestructura de comunicación, los requisitos y desarrollo del producto). Se trata de un check-list de 83 preguntas de evaluación binaria (escala Síl No) permitiendo evaluar las cuatro dimensiones estructurales. Los resultados de esta evaluación se transcriben en el instrumento de la fase 3 .

2- Una Matriz de Métodos permite identificar los medios que deberá utilizar la empresa para optimizar la implementación de la IC. Se trata de una tabla de madurez que propone métodos correlados a cada ítem de las cuatro dimensiones estructurales según niveles de acercamiento o enfoque de producto (de Tarea, de Proyecto, de Programa, de Empresa).

3- Un Mapa de Dimensiones permite representar gráficamente la diferencia o margen existente entre el estado actual del proceso y el proceso de alcanzar, mediante un gráfico sistémico ilustrando los 83 criterios de la encuesta de valoración y los niveles progresivos de los distintos enfoques de producto.

4- Un Mapa de Dimensiones de Prioridad permite equilibrar las distintas dimensiones y definir las prioridades para la implementación de la IC en la empresa, mediante una evaluación de los criterios críticos.

Si bien el modelo propuesto por Carter y Stiwell es seguramente uno de los más pertinente para el objetivo de este estudio en cuanto a la metodología propuesta, los ítems o áreas de análisis auscultados así como los niveles de madurez y, por consecuencia, los métodos propuestos para optimizar los procesos existentes no son necesariamente adaptados a las realidades de las PyMEs chilenas manufactureras.

De manera general, los instrumentos aquí analizados para mejorar los procesos de diseño y desarrollo de productos permiten, de cierta manera, codificar las buenas prácticas para llegar a metas determinadas. Sin embargo, los códigos empleados deben poder ser adaptados a las realidades de las empresas para tener efectividad.

A pesar de esto, los análisis anteriores que permitieron establecer ciertos patrones de comportamiento de las PyMEs en cuanto a sus PDDP en base a la definición de los requerimientos o criterios de implementación para el enfoque concurrente propuestos anteriormente permiten identificar una hipótesis de modelo de evaluación y mejoramiento para las PyMEs chilenas a fin de asegurar la transferibilidad del enfoque concurrente.

\section{Hipótesis para el modelo de mejoramiento de los PDDP de las PyMEs chilenas}

Los primeros diagnósticos de las PyMEs contrastados con los análisis anteriores de modelos de implementación permiten emitir algunas reflexiones en vista a proponer un modelo de evaluación y mejoramiento de los PDDP de las PyMEs chilenas, que asegure la transferibilidad del enfoque concurrente.

Primero, y al igual que Nicolini y sus colaboradores para las PyMEs argentinas, podemos establecer, en un primer análisis, que las empresas chilenas estudiadas no responden a ninguna metodología, y más aún, los patrones de comportamiento no indican que al establecer criterios estratégicos de planificación objetivos la implementación de nuevos procedimientos podría resultar durable en este tipo de empresas.

Por otra parte, y como lo vimos anteriormente, varios trabajos permiten afirmar que la dimensión cultural es la más problemática en cuanto a la necesidad de comunicación y transferencia de información que supone el enfoque concurrente [14-15, 20, 22]; pero también en cuanto a las lógicas de procedimiento $[20,10]$.

"En referencia a la gestión empresarial, la IC está representando una ruptura con las prácticas del pasado. Así, la importancia de la IC surge del hecho que desafía, no tan sólo al proceso tradicional de desarrollo de producto, sino también a las estructuras de organizaciones basadas en departamentos funcionales, a la concentración de toma de decisiones en la cúpula directiva (...)" [20]. 
Al igual que Maier, Eckert y Clarkson [16], la hipótesis de la investigación presentada es el rol fundamental que juega la comunicación en el proceso de diseño y desarrollo de productos, y que está estrechamente relacionado a la problemática cultural. Para numerosos autores, la problemática comunicacional es fundamental en la implementación de la IC, y para el éxito o falla de un proyecto de diseño y desarrollo de productos [16].

En particular, la comprensión del PDDP como proceso complejo social y técnico implica entender estos procesos no solamente desde la gestión sino que sobre todo desde una perspectiva cognoscitiva centrada en la gestión del conocimiento en las empresas. Entendiendo que la innovación que permite el proceso de DDP y que necesitan las empresas para desarrollarse se basa sobre una buena administración de los conocimientos de la empresa, el aprovechamiento de los conocimientos tácitos presentes y el fomento de las capacidades de aprendizaje para acumular experiencia y sistematizar los procesos.

Esta perspectiva cognitiva parece ser la más adecuada para abordar la problemática cultural y de gestión del cambio que se desprende de los varios diagnósticos de las PyMEs.

En particular, una de las hipótesis que se establecen para la construcción de un modelo de evaluación y mejoramiento de los PDDP de las PyMEs chilenas, basado en un enfoque concurrente es que una de las necesidades principales de estas empresas para "acercarse" [4] al modelo concurrente es la formalización de los nudos de información existentes tanto a nivel del proceso como a nivel de producto. Asimismo, el modelo que se vislumbra, a diferencia de los modelos presentados anteriormente que se enfocan sobre resultados en cada etapa del proceso [18] o sobre metas de planificación [15, 1, 4], deberá poder trabajar los mecanismos de construcción de la interdisciplinariedad, de la simultaneidad en la organización, mediante la formalización de conocimientos tácitos, muchas veces ya presente en las empresas observadas pero no racionalizados.

Efectivamente, como se constató anteriormente en un primer diagnóstico de las empresas chilenas, la mayoría practican cierto tipo de concurrencia.
Sin embargo, la informalidad de estos procedimientos impide un buen aprovechamiento de los saberes, y, por tanto, una optimización de los procesos y de los productos realizados.

Por otra parte, considerando desde un punto de vista socioantropológico la transferencia de conocimiento que genera la implementación del enfoque concurrente, así como la gestión del cambio cultural que esto supone, se plantea la hipótesis de que el modelo propuesto debe ser un instrumento basado en la autoevaluación guiada de las empresas, como lo puede proponer instrumentos basados en maturity grid. Sin embargo, el establecer niveles de madurez correspondiendo a calidad de resultados de obtener que fomenten estas tablas no resuelve la problemática de gestión del cambio. Es así que las conclusiones de esta primera fase de estudio llevan a postular por un modelo de tipo auditoría con tabla de madurez, a fin de desarrollar las capacidades necesarias para la implementación de la concurrencia; pero en las cuales los niveles de madurez deben ser definidos por los propios actores de la empresa a fin de generar los compromisos necesarios y fomentar en cada actor una apropiación del PDDP. De esta forma, y de acuerdo a las conclusiones de Wilson et al., se cree que se puede asegurar una transferibilidad del enfoque concurrente.

\section{CONCLUSIONES}

Este artículo presentó los primeros resultados de un estudio cuyo objetivo general es el desarrollo de un modelo de evaluación y mejoramiento de los PDDP en las PyMEs chilenas, basado en el enfoque concurrente.

Esta primera fase permite identificar los criterios de transferibilidad del enfoque concurrente hacia las PyMEs chilenas, puesto que un análisis de la literatura ha mostrado que no existen pruebas significativas que permitan asegurar la aplicación del modelo de IC a pequeñas y medianas empresas. Sin perjuicio de lo anterior, se han identificado varios trabajos que permiten, por una parte, apoyar el análisis de los PDDP en este tipo de empresas, y así identificar características de estas empresas, más allá de la variabilidad de sus contextos; por otra parte, analizar los modelos de implementación propuestos para las PyMEs. 
Estos dos ejes de análisis permitieron establecer criterios de transferibilidad, que se basan en dos ítems. El primero consiste en identificar las capacidades que deben desarrollar estas empresas para acercarse a un enfoque concurrente. Estas capacidades fueron establecidas mediante el análisis de las características de los PDDP de las PyMEs, y deberán ser validadas por un análisis más profundo de la recolección de datos que se realizó en tres empresas chilenas.

El segundo dice relación con el tipo de modelo de intervención de los PDDP, que según análisis de los modelos existentes, se plantea debería seguir un enfoque de gestión del conocimiento, y más particularmente, debería inspirarse del modelo de las Maturity Grid según una modalidad de autoevaluación de los procesos que permitiría fomentar los compromisos y la apropiación por los actores de la empresa de los procesos de diseño y desarrollo de producto, como mecanismo para gestionar el cambio cultural necesario a la implementación del enfoque concurrente.

Los pasos siguientes deberán, una vez validados los criterios de transferibilidad, generar una primera versión del modelo propuesto que se validará mediante consultas iterativas a las empresas estudiadas. Una segunda versión del modelo así obtenida deberá luego ser validada con un muestreo mayor de empresas.

Finalmente, se considera que esta primera fase de estudio para llegar a un modelo que permita a las PyMEs chilenas evaluar y mejorar sus PDDP es, por una parte, una necesidad para estas empresas, y para el fomento de una política de Innovación para la competitividad, por otra, permite avanzar a nivel científico, en la aplicabilidad de la IC en pequeñas y medianas empresas.

\section{AGRADECIMIENTOS}

Este artículo se realizó en el marco del proyecto DICYT 060973B: "Desarrollo de un modelo de evolución y mejoramiento de los procesos de diseño de productos para las PyMEs chilenas, basado en la ingeniería concurrente", apoyado y financiado por la Dirección de Investigación Científica y Tecnológica de la Universidad de Santiago de Chile.

\section{REFERENCIAS}

[1] F. Aguayo González y V.M. Soltero Sánchez. "Metodología del Diseño Industrial, un enfoque desde la ingeniería concurrente". Editorial Ra-Ma. Primera Edición. Madrid, España. 2002.

[2] S.L. Albin and P.J. Crefeld. "Getting started: Concurrent engineering for a medium-sized manufacturer". Journal of Manufacturing Systems. Vol. 13, Issue 1, pp. 48-58. 1994.

[3] H. Ahuett. "Evolución de las metodologías de apoyo a la ingeniería concurrente". Ingeniería Concurrente. Ediciones UPC, pp. 77-87. 2006.

[4] D.E. Carter and B.S. Baker. "Concurrent engineering: The product development Environment for the 1990s". Addison Wesley Publishing Company, Inc., 175 p. Reading, MA, USA. 1992.

[5] R. De Castro y G. Gimenez. "Evolución de la organización hacia la ingeniería concurrente". Ingeniería Concurrente. Ediciones UPC, pp. 149-159. 2006.

[6] T. Christensen and S. Yasar. "Paradigms and protocols in the study of creative collaboration: implications for research of design team process and product". IASDR07. Internacional Association of Societies of Desing Research. The Hong Kong Polytechnic University, 10 p. Hong Kong, China. November, 2007.

[7] E. Cosnard. "Problématique du déploiement de l'ingénierie simultanée concourante en PME-PMI $=$ Deployment problematics on concurrent engineering in small and medium enterprise". MICAD, pp. 171-178. Paris, Francia. 2000.

[8] N. Cross, H. Christiaans and K. Dorst. "Analysing Design Activity". Wiley. Chichester, England. December, 1996.

[9] M.R. Cutkosky and J.M Tenebaum. "Toward a Framework for Concurrent Design". International Journal of Systems Automation: Research and Applications. Vol. 1, Issue 3, pp. 239-261, 1992.

[10] A. Filson and A. Lewis. "Cultural issues in implementing changes to new product development process in a small to medium sized enterprise (SME)". Journal of 
Engineering Design. Vol. 11, Issue 2, pp. 149-157. June, 2000.

[11] V. Krishnan and K.T. Ulrich. "Product development decisions: A review of the literature". Management Science. Vol. 47, Issue 1, pp. 1-21. January, 2001.

[12] P. Lloyd, J. McDonnell, N. Cross. "Analysing Design Behaviour: The Design Thinking Research Symposia series". IASDR07. International Association of Societies of Desing Research. The Hong Kong Polytechnic University, 11 p. Hong-Kong, China. November 12-15, 2007.

[13] C. Luna Amaya, C. Berdugo Correa, M.C. Herrera Hernández y L. Prada Angarita. "Modelado del proceso de desarrollo de productos en empresas del sector metalmecánico de Barranquilla en la perspectiva de la Ingeniería Concurrente". Revista Científica Ingeniería y Desarrollo. Universidad del Norte. $\mathrm{N}^{\circ} 21$, pp. 11-25. 2007.

[14] C. Luna Amaya. "Aplicación de las técnicas y tecnologías asociadas con la ingeniería simultánea en el sector manufacturera de Barranquilla”. Revista Científica Ingeniería y Desarrollo. Universidad del Norte. $\mathrm{N}^{\circ} 8$, pp. 1-24. 2000.

[15] C. Luna Amaya y A.C. Mendoza Bayuelo "Metodología para mejorar la metodología de desarrollo de producto/proceso basada en la ingeniería concurrente". Revista Científica Ingeniería y Desarrollo. Universidad del Norte. No 16, pp. 59-69. 2004.

[16] A.M. Maier, C.M. Eckert and P.J. Clarkson. "Identifying requirements for communication support: A maturity grid-inspired approach". Expert Systems With Applications. Vol. 31, Issue 4, pp. 663-672. 2006.

[17] A. Moges Belay. "Design for Manufacturability and Concurrent Engineering for Product Development". World Academy of Science, Engineering and Technology. $\mathrm{N}^{\mathrm{o}} 49$, pp. 240246. 2009.

[18] J. Moultrie, P.J. Clarkson and D. Probert. "A tool to evaluate design performance in SMEs". International Journal of Productivity and Performance Management. Vol. 55, Issues 3/4, pp. 184-216. 2006.

[19] J. Nicolini, C. Fardelli, O. Ramírez, R. Zalazar, F. Cusolito y C. Abrevaya. "Desarrollo de productos: un análisis en PYMEs". Instituto de Industria. Universidad Nacional de General Sarmiento. Los Polvorines, Argentina. 2005.

[20] C. Riba y A. Molina. "Ingeniería concurrente - una metodología integradora". Ediciones UPC, p. 314. Barcelona, España. Octubre 2006.

[21] C. Riba Romera. "Diseño Concurrente". Ediciones UPC, 226 p. Barcelona, España. Abril 2002.

[22] J. Ríos, A. Vizán, J.M. Pérez, J.J. Márquez, J Barreiro, J. Labarga, X. Pastor and M. Montano. "Ingeniería concurrente en el diseño de moldes de inyección". Informe de proyectos: TAP-96-1453-C02-02 (CICYT), D204/1998(ATYCA-MINER). España. 1999.

[23] K.T. Ulrich, S.D. Eppinger. "Product Design and Development". Irwin/McGraw-Hill. Third Edition. New York, USA. 2004. 